SLAC-PUB-8069

April 1999

\title{
SIGNALS FROM MICROWAVE UNSTABLE BEAMS IN THE SLC DAMPING RINGS*
}

\author{
B. V. Podobedov and R.H. Siemann \\ Stanford Linear Accelerator Center, Stanford University, Stanford CA 94309
}

\begin{abstract}
The longitudinal microwave instability is present in the SLC damping rings during routine operations. Experimental studies of the instability at nominal conditions have been reported previously [1]. To complement those studies and better understand the properties of the instability a series of dedicated experiments were performed under a broad range of operating parameters. These experiments included spectral measurements of BPM signals as well as time domain diagnostics using a custom detecting circuit. This paper describes the techniques, the results and discusses possible interpretations of these measurements.
\end{abstract}

Paper Contributed to 1999Particle Accelerator Conference

New York City, New York., March 29 -April 2, 1999

* Work supported by Department of Energy contract DE-AC03-76SF00515 


\section{INTRODUCTION}

Two SLC damping rings operate to increase the brightness of $1.2 \mathrm{GeV}$ electron and positron beams before their further acceleration in the linac. Nominal store times in the rings are $8.3 \mathrm{~ms}$ for electrons and $16.6 \mathrm{~ms}$ for positrons. During normal operations both rings store two bunches each. The RF frequency is $714 \mathrm{MHz}$ and typical accelerating voltage is $V_{R F} \sim 800 \mathrm{kV}$. This results in a low current synchrotron frequency of about $\mathrm{v}_{\mathrm{s}} \sim 100 \mathrm{kHz}$ and an rms bunch length of $\sigma \sim 17$ ps. Other design and operating parameters for the SLC damping rings are described elsewhere [2].

It was reported previously [3] that above a threshold of about $2 \times 10^{10}$ particles per bunch (ppb) stored beams become longitudinally unstable. The instability develops a few milliseconds after injection which is comparable to the longitudinal damping time. Since the instability saturates quickly it does not prevent successful operation for stored currents as high as $4-5 \times 10^{10} \mathrm{ppb}$. However, it has been measured that the instability somewhat compromises the damping ring performance as an injector resulting in the beam jitter in the linac downstream [4].

The properties of instability can be summarized as follows. Instability is due to a single bunch; it contains a quadrupole or a sextupole mode and is rather sensitive to the accelerating voltage.

The simplest diagnostics for the instability is a spectrum analyzer connected to a BPM electrode. Above the threshold, high frequency revolution harmonics acquire sidebands that are displaced by roughly 2 or 3 times the unperturbed synchrotron frequency $v_{\mathrm{s}}$ indicating a quadrupole or a sextupole mode. The amplitude of the sidebands often varies slowly on a millisecond scale in a peculiar manner. This variation can be seen with a spectrum analyzer if it is set to zero span as a receiver at the sideband frequency. However, this is not a convenient measurement for longer stores when the instability sideband frequency changes as the stored charge decreases. A better way of seeing this variation is by demodulating the instability signal from the sidebands and observing the resulting low frequency signal with an oscilloscope. In addition to a slowly varying envelope this method gives a fast (roughly 2 or 3 $v_{\mathrm{S}}$ ) signal that is characteristic of the phase-space orientation of the structure associated with the instability [1].

On the other hand, dependence of the instability frequency on current as well as the amplitude dependence of a specific sideband can be measured much easier with a spectrum analyzer. Indeed, to extract the frequency from the time-domain data one needs to have large memory depth due to high ratio of the characteristic time of the envelope variation $(\mathrm{ms})$ to the instability period $(3-5 \mu \mathrm{s})$.

Although some instability properties can be measured during routine operation a dedicated experiment with a single bunch stored and flexibility in stored charge and RF voltage has more value. In this paper we report the results of a series of such experiments performed in the positron damping ring. Qualitatively similar results were obtained at a different time in the electron ring. However, since the positron ring studies were much more detailed we chose to present the positron ring results alone.

\section{SETUP AND HARDWARE}

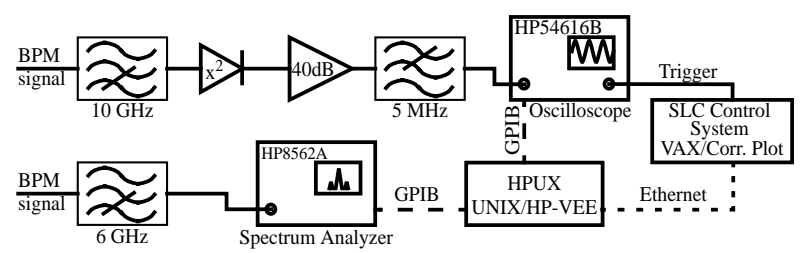

Figure 1. Experimental setup.

As shown in Fig. 1 BPM signals were simultaneously measured with two instruments i) a spectrum analyzer and ii) a digitizing oscilloscope after some initial processing. The processing circuit is described in more detail in [1]. To summarize, the front end high pass filter cuts off the bulk of the RF power coming from the BPM. A square law detector demodulates the instability signal from the sidebands to high frequency revolution harmonics. This signal is subsequently amplified, and higher order mixing products are removed by a low pass filter. During our experiments the major settings were as follows. The central frequency of the spectrum analyzer was set to the 1149th revolution harmonic which is roughly $9.77 \mathrm{GHz}$. The span was $500 \mathrm{kHz}$, and the resolution and video bandwidths were $1 \mathrm{kHz}$. This results in the sweep time of $2 \mathrm{~s}$ and, moreover, two subsequent sweeps were video averaged. The oscilloscope was AC coupled. It was set to $1 \mathrm{~ms} / \mathrm{div}$ and the trace length was 2000 points.

The oscilloscope and the spectrum analyzer were controlled through the GPIB bus from a UNIX host running HP-VEE. This program was saving the data onto the local disk and also communicated with the SLC control system VAX via Ethernet. That host was in turn running the correlation plot routine that recorded the values of the stored current as well as various RF system parameters. In addition, prior to recording each data point the control system was sending a trigger that we used for the oscilloscope. The spectrum analyzer was free running but the HP-VEE program guaranteed that the data was acquired synchronously with the oscilloscope trace. This procedure allowed us to attach the value of beam current to every pair of traces recorded. The data taking rate was a fraction of a $\mathrm{Hz}$ which was adequate for a typical beam lifetime of several minutes.

\section{MEASUREMENT RESULTS}

In the course of our measurements several dozen beam stores at various accelerating voltages were recorded. Each store lasted about seven minutes that allowed initial beam 
current of $\sim 3.5 \times 10^{10} \mathrm{ppb}$ to decay roughly a factor of two and to a value below the instability threshold. Detailed analysis of the data including the dependence of instability properties on the RF voltage will be presented elsewhere [5]. In this paper we will illustrate some of the most interesting aspects of our measurements using the data for the $\mathrm{RF}$ voltage of $\mathrm{V}_{\mathrm{RF}}=690 \mathrm{kV}$ as determined from the measured synchrotron frequency.

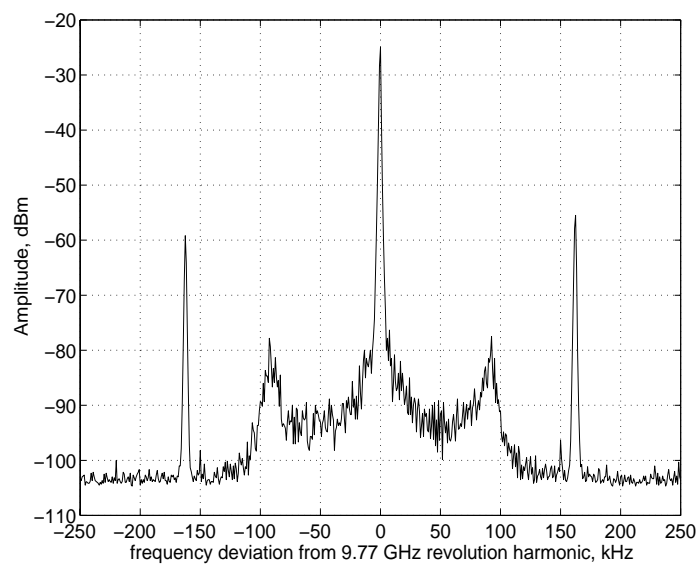

Figure 2. Typical spectrum at $\mathrm{N}=3 \times 10^{10} \mathrm{ppb}$.

The typical spectrum analyzer sweep when the quadrupole mode of the instability is present is shown in Fig. 2. One can clearly see the instability sidebands to the revolution harmonic displaced by about $160 \mathrm{kHz}$. This is roughly $10 \%$ lower than twice the zero current synchrotron frequency at this RF voltage. To represent the change of the instability spectrum with current we use a contour plot of all the spectrum analyzer sweeps for each store as shown in Fig. 3.

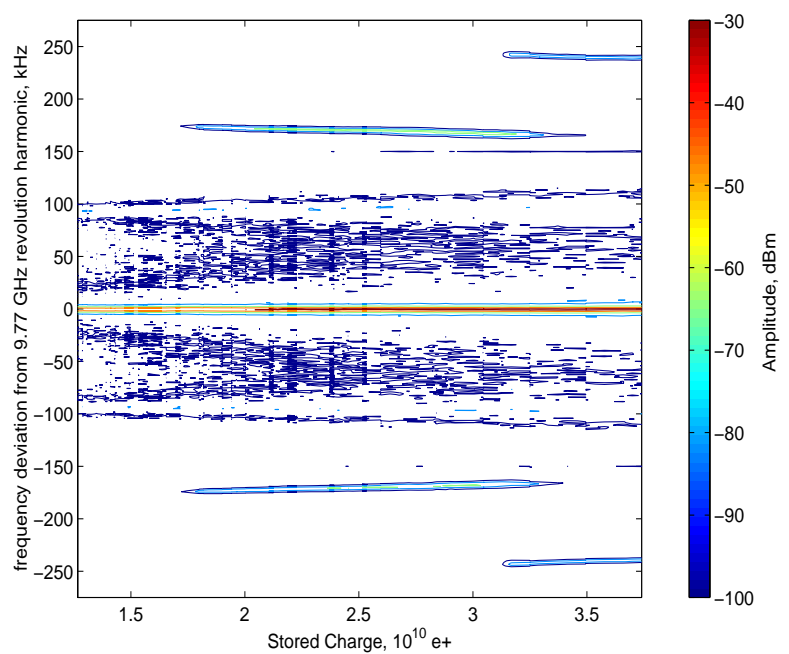

Figure 3. Spectrum analyzer data vs. stored charge.

One can see how the instability jumps from sextupole to a quadrupole mode in the region of $3.2-3.4 \times 10^{10} \mathrm{ppb}$. The quadrupole mode threshold is about $1.7 \times 10^{10} \mathrm{ppb}$. Note that other than this mode switching the instability behavior with current seems to be mundane. The fre- quency of the quadrupole mode is linearly decreasing at a rate of $\sim 5 \mathrm{kHz} / 10^{10} \mathrm{ppb}$. Such a behavior is usually attributed to the inductive portion of the ring impedance.

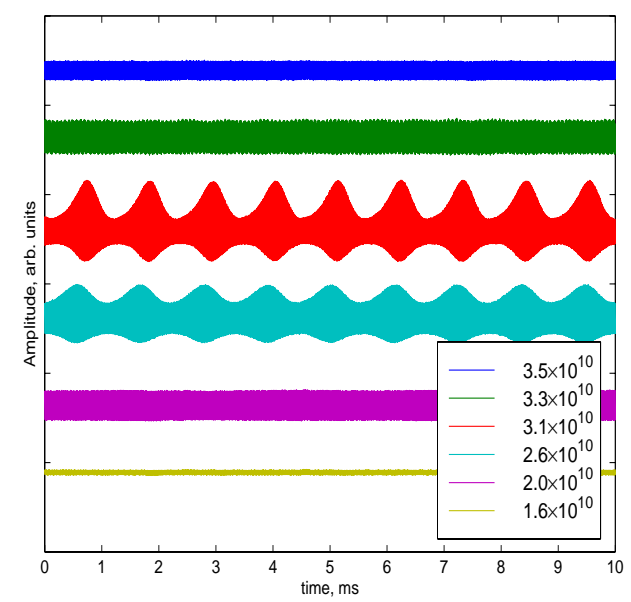

Figure 4. Oscilloscope traces of the instability signal for different values of stored charge.

The picture, however, becomes more interesting if along with the spectrum analyzer data we consider oscilloscope traces taken concurrently. Several such traces taken for the same case as Fig. 3 are shown in Fig. 4. The top trace that corresponds to the highest current shows a constant amplitude sextupole mode. The second trace from the top relates to the case when both sextupole and quadrupole modes coexist. At even smaller values of current (two traces in the middle) instability becomes pure quadrupole but it acquires the characteristic bursting behavior. As seen from Fig. 4 the bursts repeat in time with a period of about $1 \mathrm{~ms}$. Finally, below $2.5 \times 10^{10} \mathrm{ppb}$ bursts disappear and the quadrupole mode oscillates with constant amplitude.
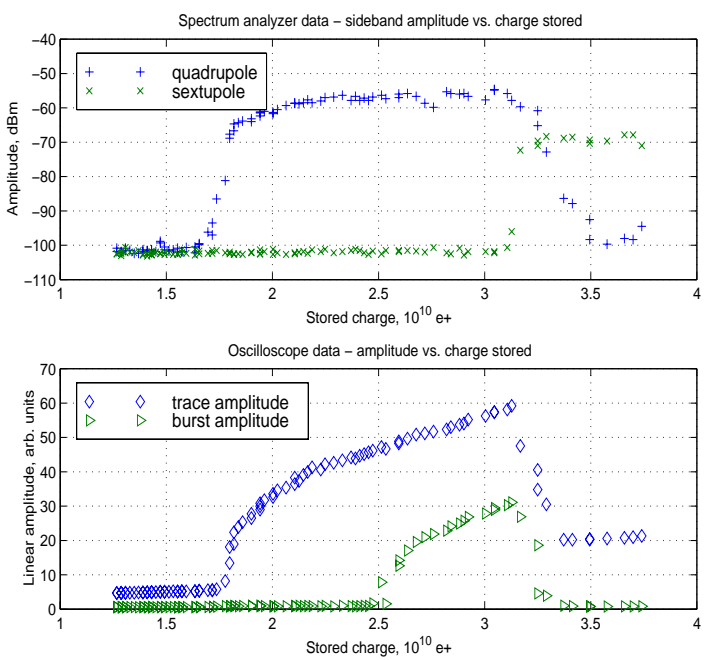

Figure 5. Instability amplitude behavior.

It is interesting to compare the amplitude dependence of the signals measured by the spectrum analyzer and the 
oscilloscope. The results for all the traces for this particular store are shown in Fig. 5. From the spectrum analyzer data we again clearly see the transition between the quadrupole and sextupole mode. It appears that the sextupole mode has a clear threshold of $3.2 \times 10^{10} \mathrm{ppb}$ while the quadrupole mode makes a slower transition in that region. In order to summarize the oscilloscope data we derived the amplitude information from the traces by simply taking the standard deviation of the whole trace (trace amplitude) and its envelope (burst amplitude). Although the oscilloscope data does not directly distinguish the two modes, the transition between them is clearly seen as the amplitude jump on the upper trace. The lower trace shows the region where instability is bursting. Note, that the thresholds seen from oscilloscope data slightly exceed the ones observed with the spectrum analyzer which can be attributed to better sensitivity of the latter. Still the agreement between the two plots in Fig. 5 is quite good.

Finally we should point out that the relative amplitudes of the quadrupole and sextupole modes seen from the spectrum analyzer or the oscilloscope data do not reflect the ratio of the quadrupole and sextupole components in the beam phase space. This is due to the fact that the amplitude of any instability sideband is not only proportional to the corresponding moment of charge density but it also has a form factor that is slowly varying with frequency. These form factors are different for each azimuthal mode and usually have the highest peak at frequency of $m /(2 \pi \sigma)$ where $m=2,3$ for quadrupole and sextupole modes respectively [6]. This is at least partially responsible for lower signal we observe for the sextupole mode that has the maximum response outside the bandwidth of the BPM pickup. One way to quantify what a particular signal means in terms of the changes to the beam phase space is to simultaneously employ a streak camera to show a variation of the beam profile with the instability signal [1].

\section{DISCUSSION}

Utilizing both frequency and time domain techniques we have developed a convenient way to characterize single bunch instability behavior.

It appears that some features of the data obtained e.g. frequency dependence on current or the quadrupole mode threshold can be explained in terms of conventional theory that is usually based on the linearized Vlasov equation. For example, in reference [7] simulations with a numerically obtained wake-function yielded values similar to those measured. However, some aspects of the measurement results clearly go beyond the linear theory. Those are 1) the fact that instability saturates, 2 ) occasional bursting behavior and 3) switching between the modes. There have been some theoretical models published [8-10] that could at least in principle lead to 1) and 2). It appears that item 3) is the most unusual. Indeed it is common to see more than one azimuthal mode go unstable as intensity increases.
What is surprising is that the mode that first goes unstable becomes stable again. Furthermore, this stability switch happens near the intensity value at which the other mode becomes unstable. This suggests some mechanism of coupling between these azimuthal modes. On the other hand it is commonly thought that as long as frequency shifts are small compared to $v_{\mathrm{s}}$ then different azimuthal modes are independent and instability can only arise as a result of other mechanisms e.g. radial mode coupling.

More theoretical work and computer simulations are required to understand this instability.

\section{REFERENCES}

[1] B. V. Podobedov, R. H. Siemann, Proc 1997 PAC, 1629 (1997).

[2] SLC Design Handbook, SLAC, (1984).

[3] K. Bane et al, Proc 1995 PAC, 3105 (1995).

[4] F.-J. Decker et al, Proc Linac 96, Geneva, 143 (1996).

[5] B. V. Podobedov, Ph.D. thesis, Stanford University (to be published).

[6] R.H. Siemann, AIP Conf. Proc. 184, 430 (1989).

[7] K. Bane, K. Oide, Proc 1995 PAC, 3112 (1995).

[8] R. Baartman, M.D'Yachkov, Proc 1995 PAC, 3119 (1995).

[9] S. Heifets, Phys. Rev. E, 54, 2889 (1996).

[10] G. Stupakov et al, Phys. Rev. E, 55, \#5, (1997). 